\title{
Electroporation of cell membranes
}

Tian Y. Tsong
Department of Biochemistry, University of Minnesota, St. Paul, Minnesota 551 8 USA

ABSTRACT Electric pulses of intensity in kilovolts per centimeter and of duration in microseconds tö milliseconds cause a temporary loss of the semipermeability of cell membranes, thus leading to ion leakage, escape of metabolites, and increased uptake by cells of drugs, molecular probes, and DNA. A generally accepted term describing this phenomenon is "electroporation." Other effects of a high-intensity electric field on cell membranes include membrane fusions, bleb formation, cell lysis . . etc. Electroporation and its related phenomena reflect the basic bioelectrochemistry of cell membranes and are thus important for the study of membrane structure and function. These phenomena also occur in such events as electric injury, electrocution, and cardiac procedures involving electric shocks. Electroporation has found applications in: (a) introduction of plasmids or foreign DNA into living cells for gene transfections, (b) fusion of cells to prepare heterokaryons, hybridoma, hybrid embryos . . etc., (c) insertion of proteins into cell membranes, $(d)$ improving drug delivery and hence effectiveness in chemotherapy of cancerous cells, $(e)$ constructing animal model by fusing human cells with animal tissues, $(f)$ activation of membrane transporters and enzymes, and $(g)$ alteration of genetic expression in living cells. A brief review of mechanistic studies of electroporation is given.

\section{ELECTRIC BREAKDOWN OF LIPID BILAYERS AND CELL MEMBRANES}

Under normal physiological conditions, a bilayer lipid membrane (BLM) made of lipid extracts of cells is a good barrier for ions and hydrophilic molecules. The membrane specific conductance, $G_{\text {membr }}$, to $\mathrm{Na}^{+}$or $\mathrm{K}^{+}$is typically $10^{-8} \mathrm{~S} \mathrm{~cm}^{-2}$ or smaller (1). For cell membranes this value is generally higher, but seldom exceeds $10^{-3} \mathrm{~S}$ $\mathrm{cm}^{-2}(1,2)$. This permeation barrier is readily modified by varying ionic compositions, adding organic compounds or detergents, or by imposing a transmembrane electric potential $(1,2)$. When an intense transmembrane electric field, exceeding the dielectric strength of a cell membrane, is applied, the membrane specific conductance increases dramatically and it can reach as high as 1 $\mathrm{S} \mathrm{cm}^{-2}$ in microseconds $(3,4)$. The phenomenon of the electric modification of cell membrane conductivity has been known since the 1940's $(1,2)$. Goldman (5) measured the voltage-current (V-I) characteristics of the membrane of Chara australia and found a phenomenon similar to the dielectric breakdown of cell membrane, i.e., an abrupt increase in the membrane conductance when the membrane was hyperpolarized beyond a certain potential. This effect was reversible: repetitive voltage scans did not alter the V-I characteristics of the membrane. Coster called it the reversible electric punch through (6). Irreversible electric breakdown of BLM and cell membranes have also been noted and the dielectric strengths determined for BLM of various lipid compositions $(1,7)$. It was found that for BLM the breakdown potential is in the range $150-500 \mathrm{mV}$ when the field duration is in microseconds to milliseconds. This value translates into a dielectric strength of $300-1,000 \mathrm{kV} / \mathrm{cm}$ when the thickness of the bilayer is $5 \mathrm{~nm}$. Cell membranes can sustain as much as $1 \mathrm{~V}$ of $\Delta \psi_{\text {membr, }}$ i.e., an electric field strength of $2,000 \mathrm{kV} / \mathrm{cm}$, when microsecond to millisecond electric pulses are used $(8,9)$. The dielectric strength of cell membranes depends both on the amplitude and on the length of the applied electric field (10). Despite much information which had already been obtained in these early studies concerning effects of electric field on membrane permeability, their influence on the later development of the discipline now identified as the study of electroporation of cell membranes and its related phenomena remains unclear. Many investigators working on mechanisms of electroporation and applications of these techniques in biotechnology would recognize the pioneering contributions of $\mathrm{H}$. Schwan (11) and the late $\mathrm{H}$. Pohl (12). However, a notable surge of activity in the last 10 years must be attributed to the success of using the pulsed electric field (PEF) method for loading exogenous molecules $(13,14)$, gene transfer $(15,16)$, and cell fusions $(10,17-20)$.

\section{TIME SEQUENCE AND MOLECULAR MECHANISMS OF ELECTROPORATION}

\section{Lipid bilayer}

Two properties of a lipid bilayer would render it susceptible to influence by an applied electric field: the charges or the electric dipoles of the lipid molecule and the small 
but nevertheless finite permeability of the bilayer to ions. The former would cause lipid molecules to reorient under an intense electric field, thus, creating hydrophilic pores and impairing the bilayer to serve as a barrier against ions. The latter would conduct current, thus, generating local Joule heating and inducing thermal phase transitions of the lipid bilayer. Molecular dynamics of these events, either of the thermal origin or of the electrical origin, would be similar, both involving changes in conformation of lipid molecules and rearrangement of the bilayer by first, expanding the existing, or creating new hydrophobic pores and second, forming structurally more stable hydrophilic pores. The apparent permeability, $P_{\text {app }}$, of a lipid bilayer under conditions where the gel state (S-state) and the liquid-crystalline state (F-state) coexist may be conveniently described by Eq. $1(21,22)$.

$$
\begin{aligned}
P_{\text {app }}(T)= & P_{\mathrm{S}} \alpha_{\mathrm{S}} \exp \left(-E_{\mathrm{S}} / R T\right) \\
& +P_{\mathrm{F}} \alpha_{\mathrm{F}} \exp \left(-E_{\mathrm{F}} / R T\right)+P_{\mathrm{B}} \alpha_{\mathrm{B}} \exp \left(-E_{\mathrm{B}} / R T\right) .
\end{aligned}
$$

In the equation, $P_{\mathrm{i}}, \alpha_{\mathrm{i}}$, and $E_{\mathrm{i}}$ are, respectively, the permeability of lipid bilayer, the fraction of lipid, and the temperature dependence term (Arrhenius activation energy) of the permeability in the S, F, or B-phase. The B-phase has been defined either as the interface of lipid clusters in the S- and the F-phases (21), or as a parameter which measures fluctuation properties such as the compressibility, the heat capacity ... etc. of the domain interfaces $(22,23)$. The permeability of $\mathrm{Na}^{+}, \mathrm{K}^{+}$, and other hydrophilic molecules have been found to exhibit a pronounced maximum close to the phase transition temperature of lipid vesicles $(21,24)$, which is reflective of the property of the parameters $\alpha_{B}(21,22)$. It is concluded that the transbilayer pathway of ionic species occurs preferentially at the boundary regions of the lipid cluster (21). In other words, for ions, $P_{\mathrm{B}} \gg P_{\mathrm{S}}$, $P_{\mathrm{F}}$. These boundary regions may be considered defects of the crystal lattices. Lipid molecules in these regions lack regular cooperative interactions with their nearest neighbors and are least restricted for motions. Solvent molecules and hydrated ions are most likely to permeate these fluctuating defects. However, for nonpolar species, $P_{\mathrm{F}}>P_{\mathrm{S}}$, and $P_{\mathrm{B}}=0$, and for neutral species, $P_{\mathrm{F}}>$ $P_{\mathrm{S}}>P_{\mathrm{B}}(21)$.

These studies show that the conductance of ions through a lipid bilayer mainly occurs at lattice defects. However, lattice defects are not static structures. They are constantly fluctuating in the time scales of picoseconds to minutes (25-27). The formation of a kink in a lipid molecule (or the trans to cis transition of a $\mathrm{CH}_{2}-\mathrm{CH}_{2}$ bond) occurs in $5 \mathrm{~ns}$ (28), and the propagation of kinked conformation to its neighboring molecules occurs in 20-200 $\mu \mathrm{s}$. After these rapid events, there are slow, highly cooperative structure rearrange- ment in milliseconds to minutes (25-27). Rotation of lipid molecules occurs in microseconds. The lateral diffusion coefficient of lipid is $\sim 1 \times 10^{-8} \mathrm{~cm}^{-2} \mathrm{~s}^{-1}$ (29, 30 ) and the transbilayer flip-flop of lipid is an extremely slow process, with a time counstant of hours to days (30). These time constants are measured in the absence of an applied electric field and reflect different modes of thermal motions of molecules. In the presence of an electric field, a rate for orientation of an electric dipole will be enhanced according to Eq. 2 (31).

$$
k_{\text {elec }}=k_{0} \exp \left[-r \Delta M_{\text {elec }} \cdot E_{\text {membr }} / R T\right],
$$

where $k_{0}, k_{\text {elec }}, r, \Delta M_{\text {elec }}, E_{\text {membr }}$, and $R$ are the rate constant under zero field, under an electric field, the apportionation constant (between 0 and 1 ), the difference in molar electric moments of the molecule, the electric field strength across the lipid bilayer, and the Gas Constant, respectively. Kinetics of electroporation of BLM and cell membranes have been measured by the chargepulse relaxation method $(32,33)$ or by the conductivity measurement (4). The breakdown of BLM occurs in submicrosecond time ranges. The generation of $\Delta \psi_{\text {membr }}$ in a large lipid vesicle $(\sim 40 \mu \mathrm{m})$ depends on salt concentration and is in the microsecond time range $(K$. Kinosita, personal communication). Theoretically, the $\Delta \psi_{\text {membr }}$ induced by a step function electric field depends on the radius of the lipid vesicle, $R_{\text {cell }}$, the specific resistivities of the internal $\left(r_{\text {int }}\right)$, and the external $\left(r_{\text {ext }}\right)$ media $(3,4,10)$.

$$
\Delta \psi_{\text {membr }}=1.5 R_{\text {cell }} E_{\text {appl }} \cos \theta\left[1-\exp \left(-t / \tau_{\text {membr }}\right)\right],
$$

where $\theta$ is the angle between the field line and the normal from the center of the vesicle to a point of interest on the membrane surface. The membrane relaxation time is

$$
\tau_{\text {membr }}=R_{\text {cell }} C_{\text {membr }}\left(r_{\text {int }}+r_{\text {ext }} / 2\right) .
$$

$C_{\text {membr }}$ is the capacitance per unit area of the membrane. Since experimentally measured $\tau_{\text {membr }}$ and the fast phase of the electroporation are similar $(3,4,32,33)$, it is likely that pore initiation occurs at the existing fluctuating lattice defects of the lipid bilayer.

Once a current, $i$, is induced in a defect, $i^{2} r \Delta t$ of heat is generated, where $r$ is the resistivity of the defect, $\Delta t$, the field duration. This local heating could cause a phase transition or disordering of lipids $(21-28,34,35)$. Lopez et al. (35) have measured ${ }^{31} \mathrm{P}$ NMR of Chinese hamster ovary cells (35). A new isotropic peak was detected for the electropermeabilized cells, suggesting that electric pulses caused disordering of membrane lipids (35). Sugar has proposed a Stochastic model to explain field-induced pore formation (36). Weaver has calculated extent of local heating using estimated values of 
effective transmembrane electric field, conductivity of medium, and pore size (37). Local temperature rise of several degrees may be reached in microseconds to milliseconds (37). If $\Delta t$ is a few milliseconds or longer, the current could also produce electroosmosis effects (38), thus, mechanically enlarging the defect. Pores of diameters in micrometers have been observed by timeresolved fluorescence imaging of large lipid vesicles (diam $40 \mu \mathrm{m})$ (K. Kinosita, Jr., personal communication).

Based on the above observations, Scheme 5 has been proposed to describe the reversible electroporation of the lipid vesicles and for a more drastic perturbation of lipid vesicles by high-intensity electric pulses, Scheme 6 has been proposed (39).

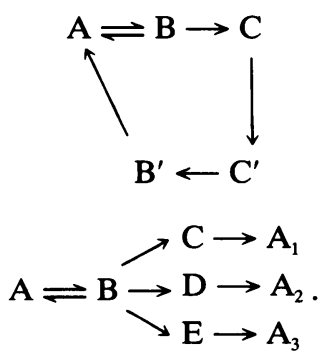

In these two schemes, the A to B transition represents the reversible pore initiation step that takes place in submicroseconds or shorter. After this step, if the applied field-induced $\Delta \psi_{\text {membr }}$ exceeds the dielectric strength of the bilayer, a pore expansion step, B to C, follows (Scheme 5). This process is considered irreversible because resealing takes seconds to minutes after the electric pulse terminates. Because the conditions for electroporation and resealing are different, the former being in the presence and the latter being in the absence of an electric field, their paths are necessarily different. $B^{\prime}$ and $C^{\prime}$ are equivalent states of $B$ and $C$, respectively, in the absence of an electric field. If the induced $\Delta \psi_{\text {membr }}$ greatly exceeds the dielectric strength of the lipid bilayer, a large vesicle may be fragmented due to the electrostatic repulsion of polarized molecules (B to C, D and $E$ transitions), the end products would be small vesicles $\left(A_{1}, A_{2}\right.$, and $\left.A_{3}\right)$.

Theoretical treatments of electroporation have considered the stability of hydrophobic and hydrophilic pores $(40,41)$. The stability of a pore depends on the pore "energy" which is the sum of mechanical energy, electrical contribution, and others (42). The mechanical term is a function of the edge energy and the surface tension of the membrane-water interface. Weaver and coworkers have used these assumptions to calculate timedependent size distributions of electropores in BLM and a model cell (42). Their results are consistent with the conductance measurements of BLM breakdown by Benz. et al. (32). Uptake of different sizes of fluorescence probes by electroporated erythrocytes and yeast has been measured using flow cytometry to test the prediction of the theoretical models (43).

\section{Cell membrane}

The opening/closing of many protein channels is known to depend on transmembrane electric potential $(44,45)$. The gating potentials of these protein channels are in the $50-\mathrm{mV}$ range. This is considerably smaller than the dielectric strength of a lipid bilayer. Thus, one may expect that when a PEF is applied, many voltagesensitive channel proteins will open before the $\Delta \psi_{\text {membr }}$ reaches the breakdown potential of the lipid bilayer, i.e., $150-500 \mathrm{mV}$. However, opening of these voltagesensitive protein channels may not be sufficient to prevent a continuous increase of $\Delta \psi_{\text {membr }}$ to reach the breakdown potential of the lipid bilayer. Protein channels once open may experience current much larger than they are designed to conduct. As a result these channels may be irreversibly denatured by Joule heating or electric modification of their functional groups. Thus, electroporation in a cell membrane can occur both in protein channels and in the lipid domain. Kinetic schemes representing the complete process are (39)

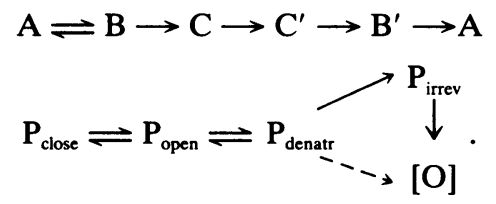

Scheme 7 is identical to Scheme 5 and describes electroporation through lipid domains of a cell membrane. In Scheme 8, a channel protein is shown to be in the closed, open, reversibly denatured, and irreversibly denatured states. [O] denotes a protein which after being irreversibly denatured by an electric pulse is excised from the membrane, e.g., by endocytosis for recycling. Reversible opening/closing of a protein channel occur in the microsecond time range. Denaturation of a protein takes place in milliseconds to seconds (46). Excision of an irreversibly denatured protein may take many minutes.

Electroporation occurring in the lipid domain is expected to reseal in milliseconds to seconds, as is the case for BLM or lipid vesicles $(47,48)$. Membrane proteins are much more complex and their dynamics cover broader time ranges (from nanoseconds to hours). Experimentally, kinetics of electroporation of cell membranes have been monitored by permeability, conductivity, and fluorescence imaging methods $(3,49-53)$. These methods detect events of Schemes 7 and 8 . In one case, $\mathrm{Na}, \mathrm{K}-\mathrm{ATPase}$ of human erythrocyte is shown to respond to $\mathrm{PEF}$ and generate transmembrane current which can 
be inhibited by ouabain, the specific inhibitor of the enzyme (54).

For cell membranes, there are other secondary effects of an electric field which are not represented by these kinetic schemes. The molecular events detected by rapid freezing electron microscopy are not in complete accord with those detected by conductivity measurement. With conductivity measurements, a submicrosecond pore initiation is recorded which has been confirmed by ion leakage experiment $(4,53)$. Pores expand in the $100-\mu \mathrm{s}$ time range and begin to reseal (incompletely) within milliseconds. Electronmicroscopy does not detect porelike structures until $10 \mathrm{~ms}$ after the PEF was terminated (55). Apparently, the relatively large pore-like imprints imaged by the electronmicroscopy result from secondary effect of PEF. They may reflect reorganization of the cytoskeletal network. Loss of the natural membrane potential of a cell after electroporation may also lead to loss of lipid asymmetry or other supramolecular structures in the cytoplasm.
Large scale structural modification of cell membranes is expected for cells treated with an electric field which will generate a $\Delta \psi_{\text {membr }}$ much bigger than $1 \mathrm{~V}$ for milliseconds or longer. Membrane fragmentation such as that represented by Scheme 6 will take place. However, even with field strengths that are only slightly higher than the breakdown voltage, membrane blebs may form in certain types of cells $(56,57)$. The most prominent of the field induced secondary effect is the lysis of electroporated cells. This phenomenon has been studied in great details for human erythrocytes by Kinosita and Tsong $(4,13,49,58)$. They have found that cell lysis is due to the colloidal osmotic pressure of the cytoplasmic macromolecules. Cell lysis may be prevented by balancing the colloidal osmolality of cells with molecules larger than the size of the electropores in solution $(39,49)$. This procedure stops cells from swelling and greatly facilitates membrane resealing. Fig. 1 summarizes the main events of electroporation and resealing of cell membranes. Molecular events leading

Electroporation Osmotic imbalance Swelling Membrane rupture

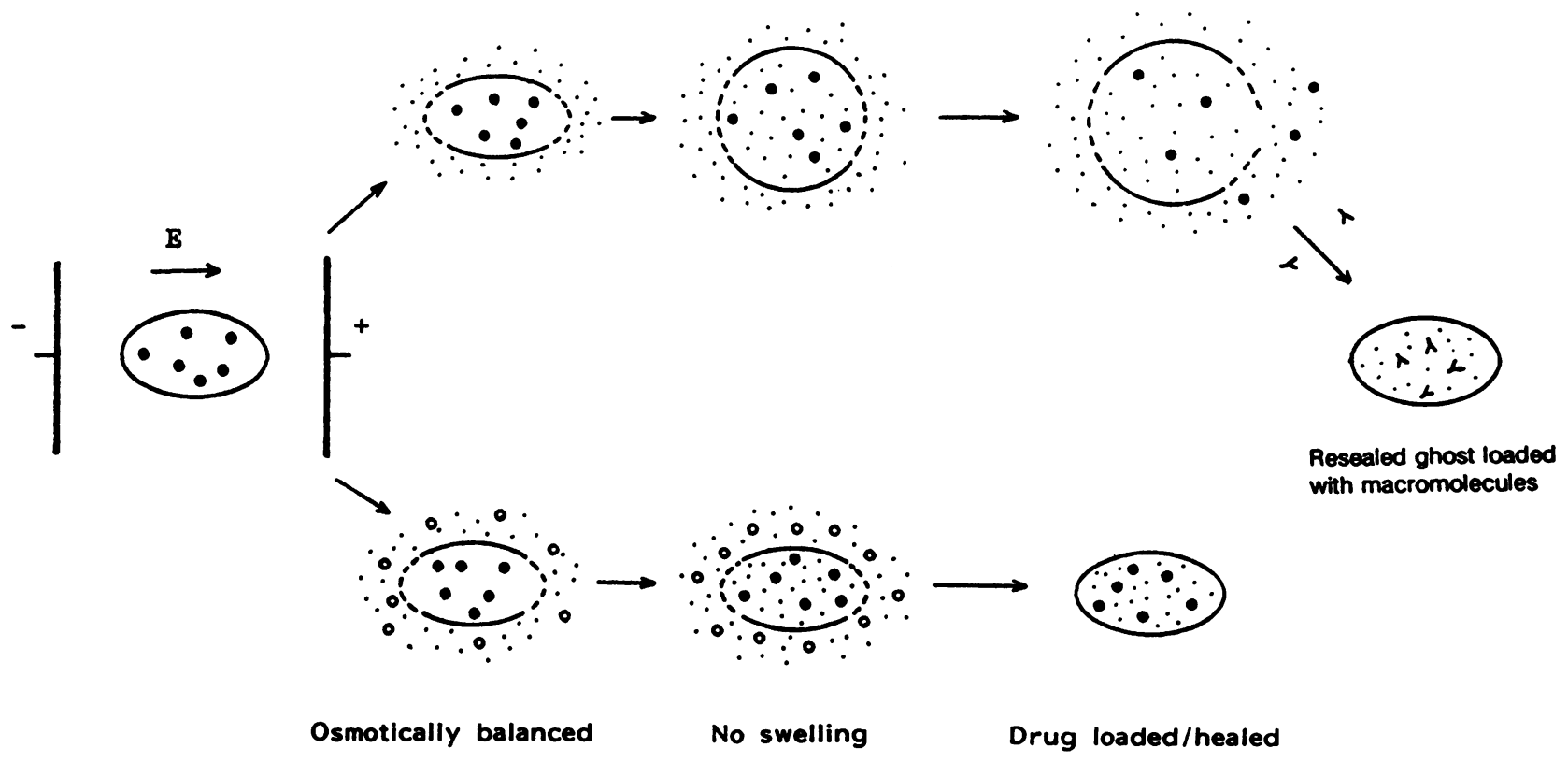

FIGURE 1 Electroporation and resealing of cell membrane. Two paths for loading of exogenous molecules and cell recovery are compared. In the upper path, after the electroporation, the plasma membrane becomes permeable to small size molecule (dots) but not the cytoplasmic macromolecules (small dark circles). The colloid osmotic pressure of cellular macromolecule would drive the influx of water and small solutes. The cell swells and eventually the cell membrane ruptures leading to cell lysis. Thereafter, the cell envelope or ghost deflates and reseals slowly. At the cell lysis and deflating step, macromolecules such as enzymes or antibodies may be loaded. The final product is the cell ghost loaded with drugs and biologically active macromolecules. In the lower path, a molecule larger than the size of electropores (small open circles) is added to counter balance the colloidal osmotic pressure of the cytoplasmic macromolecules. Cell swelling is prevented and the membrane reseals. The final product is the drug loaded cell, which is viable and indistinguishable from the untreated cell except it now carries a drug. See text for details. 
to the complete resealing of electroperforated cell membranes remain to be investigated.

Until now very little was known about the effects of a PEF on the chemistry of cell membranes. The generation of a $\Delta \psi_{\text {membr }}$ will inevitably alter the charge distribution of certain proteins either by electroconformational change $(31,39)$ or by changes in the phosphorylation of amino acids. A PEF may also cause redox reactions, e.g., oxidation of $-\mathrm{SH}$ groups or methionine etc. Byproducts of an electric pulse may also interfere with the normal function of a cell membrane, or prevent a porated membrane from resealing. Work by Deuticke and coworkers are aiming at answering these questions (59, $60)$.

\section{Electroporation by oscillating electric fields}

Eq. 4 shows that the relaxation time of a membrane, $\tau_{\text {membr }}$, is the rise time of $\Delta \psi_{\text {membr }}$. If the frequency of an applied ac field, $f_{\text {ac }}$, approaches $1 / \tau_{\text {membr }}$, the induced $\Delta \psi_{\text {membr }}$ will be smaller than that predicted for a dc field, Eq. 9. In such a case, the Schwan Equation, Eq. 10, should apply (61-63).

$$
\begin{gathered}
\Delta \psi_{\text {membr,dc }}=1.5 R_{\text {cell }} E_{\text {appl }} \cos \theta \\
\Delta \psi_{\text {membr,ac }}=1.5 R_{\text {cell }} E_{\text {appl }} \cos \theta /\left[1+\left(2 \pi f_{\text {ac }} \tau_{\text {membr }}\right)^{2}\right]^{1 / 2} .
\end{gathered}
$$

For an ac, $E_{\text {appl }}=E^{0} \sin 2 \pi f_{\mathrm{ac}} t, E^{0}$ being the amplitude of the ac field. For biological cells of diameters in micrometers, $\tau_{\text {membr }}<1 \mu \mathrm{s}$. Cells of larger diameters have $\tau_{\text {membr }}>$ $1 \mu \mathrm{s}$.

Pliquett (56) has reported that an ac field can cause membrane morphological changes and eventually lysis of cells. Chang and co-workers have used dc shifted oscillating electric fields for electroporation and electrotransfection of cells by plasmid DNA $(55,64)$. The intensities of ac fields employed in these experiments (kilovolts per centimeter range) are comparable to those of dc pulses for inducing similar reactions. If $f_{\mathrm{ac}}$ is smaller than $100 \mathrm{kHz}, \Delta \psi_{\text {membr,dc }} \approx \Delta \psi_{\text {membr,ac }}$, these PEF-related phenomena should be mechanistically similar. However, ac fields of much weaker intensity, e.g., $\sim 20-200 \mathrm{~V} / \mathrm{cm}$ have been shown to change membrane conductance and facilitate transfection of Escherichia coli by plasmid DNA (65). In these low-intensity ac experiments, effects of fields are reversible in $<1 \mathrm{~min}$, contrary to the recovery time of the high field experiment which is between $10 \mathrm{~min}$ and an hour. The low amplitude ac-treated cells are completely viable. Electroconformational coupling of membrane transport systems has been invoked to interpret these results (65).

\section{SOME RELATED PHENOMENA}

\section{Electrofusion of cell membranes}

Application of electric pulses to the suspension of lipid vesicles or cells may cause fusions of these vesicles and cells. The phenomenon was thought to result from thermal melting and merging of lipid bilayers caused by Joule heating of a PEF (66). It is now generally accepted that electrofusion of cell membranes is due to nonthermal effects of a PEF and may be related to electroporation of cell membranes $(10,17-20)$. Although electrofusion is emerging as a popular method in hybridoma technology, genetic engineering, and agricultural research, not much is known of the molecular mechanism of electric membrane fusion. A PEF can modify a cell membrane in many different ways, the most prominent are disordering of the lipid bilayer and transient changes in cytoskeletal structures. Direct links between these events and membrane fusion, however, are still lacking. Hermann et al. (67) in a recent study observe that loss of lipid asymmetry in erythrocyte ghosts greatly facilitates fusion of vesicular stomatitis virus. Loss of lipid asymmetry could be the "fusogenic state" proposed by Sowers (68). Heterokaryons or hybridoma produced by electrofusion are viable but cells losing lipid asymmetry may not be viable (69). These conflicting observations would suggest that other effects of a PEF may have to be considered.

Electrofusion is not a one-step process. Several intermediate products have been identified. Fusion that involves only the merging of the outer monolayer of the lipid bilayer will allow diffusion of fluorescence lipid probes but not the mixing of the cytoplasmic content. Content mixing is a necessary criterion for a complete fusion of two cells $(70,71)$. Fig. 2 summarizes different pathways of electrofusion of cells.

\section{Electrotransfection}

Wong and Neumann (15) and Neumann et al. (16) were the first to report successful introduction of the thymidine kinase (tk) gene into tk-deficient cultured mouse L-cells by the electroporation method and to demonstrate the expression of the loaded genes. Electrotransfection has become a routine technique for DNA transfection of cells. In most experiments plasmid DNA are used. These DNA have high molecular weight and it is not clear how such macromolecules can enter a cell through electropores. Except for the micrometer size volcano-like structures seen in the replica of the frozen erythrocyte samples (55), most estimates by measurement of the permeability of membrane probes give the 


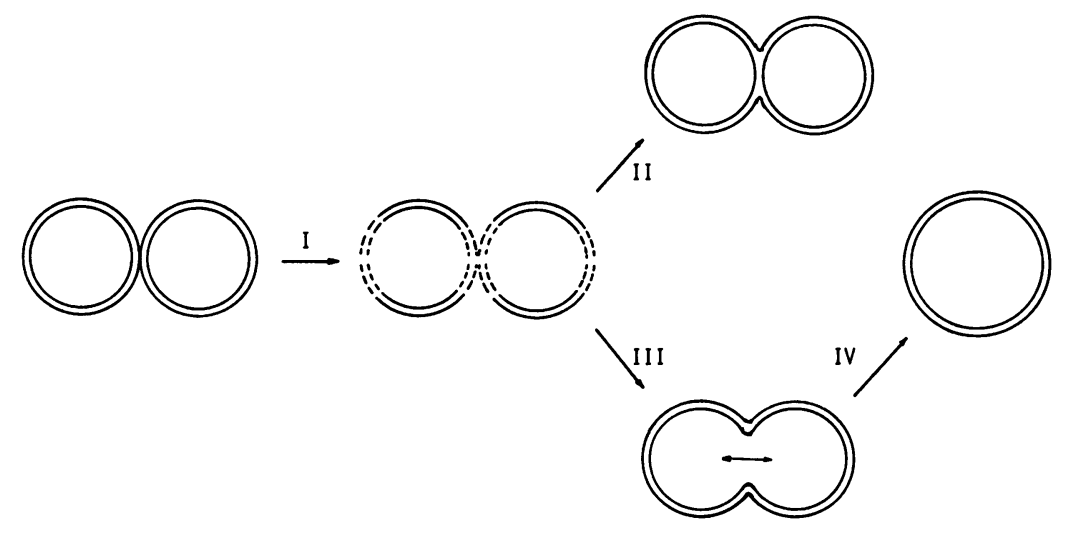

FIGURE 2 Different pathways for electrofusion of cells. After the electroporation of two cells in contact (Step I), fusion of membranes may occur only for the outer monolayers of the lipid bilayers (Step II). The end product will be a stable conjugate of cells, adjoined by a common envelope but with no mixing of the cytoplasmic contents. If fusion occurs in both monolayers of the lipid bilayer (Step III), the cytoskeletal networks of the two cells will slowly merge (Step IV). The end product will be a completely fused cell with a shared cytoplasm.

size of "stable" electropores to be $\sim 1-10 \mathrm{~nm}(3,52,72$, 73), which would be too small to permit DNA entry. Chizmadzhev and co-workers (74) propose that electrophoretic force of the PEF is responsible for driving DNA into a cell. Chernomordik et al. (74) have shown that DNA taken up by lipid vesicles are enclosed by a shell of lipids. In other studies, it has been shown that only the DNA which is surface bound is able to transfect $E$. coli after electric pulse treatment (75). DNA transfection has also been shown to occur using ac fields of strength less than one-tenth that required for electroporation (65). Fig. 3 compares two plausible mechanisms of DNA transfection.

\section{Electroinsertion of membrane proteins}

Purified cloned membrane proteins may be correctly incorporated into cell membranes by using the PEF method. Mouneimne et al. (76) have used this technique to incorporate human glycophorin into mouse erythrocyte membranes and the full length recombinant CD4 receptor into human and murine red blood cells (77). CD4 is the receptor of gp120, an HIV virus infecting protein. Besides its potential for clinical applications, such as these studies, the electroinsertion promises to be an effective and convenient means for the reconstitution of membrane proteins into lipid vesicles and cell mem-

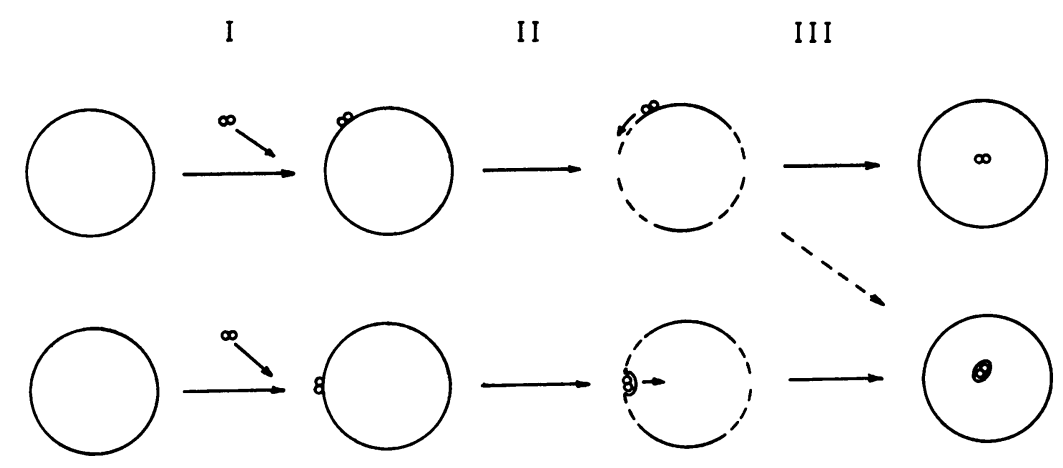

FIGURE 3 Two mechanisms for electrotransfection of cell by plasmid DNA. In the upper path, DNA binds to the cell surface in Step I. After electroporation (Step II), DNA diffuses into the cytoplasm leading to the transfection of the cell (75). In the lower path, surface-bound DNA is driven into the cell by electrophoretic force of the applied field (Step II). The loaded DNA is enclosed in a shell of lipid and is not accessible to the molecular probe ethidium bromide (74). In the upper path, the uptake of surface-bound DNA may occur through an endocytosis-like mechanism. In such a case, DNA would also be protected by a lipid enclosure. 
branes. The method should have a bright future for the study of the biochemistry of cell membranes.

\section{Electroactivation and energy coupling}

If field strength is properly controlled to generate a $\Delta \psi_{\text {membr }}$ comparable to the endogenous transmembrane potential of a cell or an organelle, a PEF may induce the reversible conformational change of biologically active molecules as represented by the $A \rightleftharpoons B$ transition of Eq. 7 and the $P_{\text {close }} \rightleftharpoons P_{\text {open }}$ transition of Eq. 8. In a cell membrane, the interaction of a protein with an electric field is "vectorial" and the activation process is necessarily "anisotropic" (78). This type of interaction has been exploited to formulate mechanisms for energy and signal transductions by membrane proteins $(31,79)$. It has been shown that an oscillating electric field of appropriate amplitude and frequency can enforce and thus synchronize the conformational oscillation of a protein. If the "enforced conformational oscillation" is coupled to a ligand binding reaction or a substrate-product conversion, energy transmitted through the electric field may be captured and transferred to drive an endergonic reaction. Such a mechanism, electroconformational coupling, has been used successfully to interpret an acinduced active pumping of cations by $\mathrm{Na}, \mathrm{K}-\mathrm{ATPase}$ and ATP synthesis by mitochondrial ATPase, apparently in the absence of energy sources except the applied electric field $(79,80)$. Using the same concept, cells are shown to be more tolerant of oscillating electric fields in electrotransfection experiments (65). Electroconformational coupling has also been used to interpret how a cell or an organism can recognize very weak electric signals (81). Other driving forces, such as acoustic, mechanical, thermal energy ... etc. may also be used to enforce conformational oscillations of biological molecules for signal recognition and emission (78). An alternative model by Blank considers effects of electric fields on ionic distributions near a cell membrane (82). The activity of a membrane enzyme may depend on local concentration of an ion. The model has successfully explained their data on effects of low-intensity, lowamplitude electric fields on the ATP splitting activity of Na,K-ATPase (83).

\section{Clinical relevance}

Electroporation and electrofusion of cell membranes have been found to occur in many clinical situations. Common electrical injuries involve extensive electroporation of muscle cells and heat denaturation of muscle proteins (84). In cardiac procedures comprising electric shock, electroporation of cardiac muscle cells and the concomitant $\mathrm{Ca}^{++}$leakage is a source of clinical complication (85). The plasma membrane of most pathological cells have weakened dielectric strength. This weakened membrane greatly reduces the cells' ability to perform normal functions because of ion leakage. Lysis of such

TABLE 1 Some effects of an electric field on chemical reaction

\begin{tabular}{|c|c|c|}
\hline & Effects common to all systems & Effects specific to cells in suspension \\
\hline Electric field effects & $\begin{array}{l}\text { Electrophoresis } \\
\text { Orientation of molecules } \\
\text { Wien effect or ion pair dissociation } \\
\text { Electroconformational change } \Delta K / K= \\
\quad \Delta M \cdot \Delta E / R T\end{array}$ & $\begin{array}{l}\text { Field-induced transmembrane potential } \\
\text { Amplification of common effects within cell } \\
\text { membrane by } 1.5 R_{\text {cell }} / d \text { for a spherical cell } \\
\text { Large transmembrane current } \\
\text { Electrocompression of membrane }\end{array}$ \\
\hline For alternating field only & $\begin{array}{l}\text { Dielectrophoresis } \\
\text { Enforced conformational oscillation for } \\
\text { energy and signal transductions }\end{array}$ & $\begin{array}{l}\text { Amplification of common effects within cell } \\
\text { membrane }\end{array}$ \\
\hline $\begin{array}{l}\text { Thermal effects current effects } \Delta T= \\
i^{2} r \Delta t /\left(4.18 C_{\mathrm{p}}\right)\end{array}$ & $\begin{array}{l}\text { Enthalpy effects } \Delta K / K=\left(\Delta H / R T^{2}\right) \Delta T \\
\text { Solvent expansion or shock wave } \Delta P= \\
\quad(\alpha / \kappa) \Delta T\end{array}$ & $\begin{array}{l}\text { Amplification of common effects due to } \\
\text { transmembrane current } \\
\text { Thermal osmosis effects } \Delta \Pi=-(Q / v T) \Delta T \\
\text { Colligative effects } \Delta \Pi=c R \Delta T\end{array}$ \\
\hline
\end{tabular}

Symbols used: $R_{\text {cell }}$, radius of cell; $\Delta T$, temperature change; $r$, specific resistivity of solution; $C_{\mathrm{p}}$, specific heat capacity of solution; $c$, concentration of solute; $K$, equilibrium constant; $\Delta M$, change in the molar electric moment; $\Delta H$, enthalpy of reaction; $\Delta P$, pressure generated by shock wave; $\alpha$, thermal expansion coefficient of solvent; $\kappa$, compressibility of solvent; $Q$, heat of transfer of water across cell membrane; $d$, membrane thickness; $i$, current; $\Delta t$, field exposure time; $\Delta \Pi$, change in osmotic pressure; $R$, gas constant; $\Delta K$, change in equilibrium; $\Delta E$, effective electric field; $T$, Kelvin temperature; and $v$, partial molar volume of solvent. See references 39 and 58 for details. 
cells is likely to be due to electroporation by endogenous electric potential.

In a recent study, Mir and co-workers have applied electroporation locally to cancerous tissue to facilitate entry of bleomycin into tumor cells. The cytotoxicity of the drug increased by 650,000 -fold. A suppression of tumor growth leading to the complete recession of tumors in some experimental mice has been recorded (86). Numerous clinical applications of electroporation and electrofusion have been reported, e.g., for microsurgery, selective tissue DNA transfection, fusion of human cells to animal tissue to construct animal models, establishment of library of hybridomas ... etc. Studies of electric properties of cell membranes are also expanding into the area of environmental concerns, e.g., effects of ELF (extremely low frequency electromagnetic fields) on cell function and on health (87).

\section{PERSPECTIVE}

Studies of the electric properties of cells began before the development of the membrane hypothesis of cells (2). However, electroporation as a discipline has only 15 years of history. In this short span of time, many phenomena have already been found and studied and new phenomena are frequently being reported in the literature. This is not surprising because a PEF can exert different effects on a chemical reaction especially that occurring in a cell membrane (Table 1). Future studies shall clarify these different effects of a PEF and also focus on understanding molecular mechanisms of these PEF-induced membrane phenomena.

I thank my former and current colleagues, my collaborators, and investigators working on electroporation and electrofusion of cell membranes. Their persistence, curiosity, and scientific integrity have shaped the discipline into its current state. Thanks are also due to C. J. Gross who helped me prepare the manuscript.

Received for publication 2 January 1991 and in final form 12 April 1991.

\section{REFERENCES}

1. Tien, H. T. 1974. Bilayer Lipid Membranes (BLM): Theory and Practice. Marcel Dekker, Inc., New York. 655 pp.

2. Cole, K. S. 1972. Membranes, Ions and Impulses. University of California Press, Berkeley. 569 pp.

3. Hibino, M., M. Shigemori, H. Itoh, K. Nagayama, and K. Kinosita, Jr. 1991. Membrane conductance of an electroporated cell analyzed by submicrosecond imaging of transmembrane potential. Biophys. J. 59:209-220.

4. Kinosita, Jr., K., and T. Y. Tsong. 1979. Voltage-induced conduc- tance of human erythrocyte membranes. Biochim. Biophys. Acta. 554:1015-1019.

5. Goldman, D. E. 1943. Potential impedance and rectification in membranes. J. Gen. Physiol. 27:37-50.

6. Coster, H. G. L. 1965. A quantitative analysis of the voltagecurrent relationships of fixed charge membranes and the associated property of "punch-through." Biophys. J. 5:669-686.

7. Huang, C.-H., L. Wheeldon, and T. E. Thompson. 1964. The properties of lipid bilayer membranes separating two aqueous phases: formation of a membrane of simple composition. J. Mol. Biol. 8:148-160.

8. Sale, A. J. H., and W. A. Hamilton. 1968. Effects of high electric fields in microorganisms. III. Lysis of erythrocytes and protoplasts. Biochim. Biophys. Acta. 163:37-43.

9. Coster, H. G. L., and U. Zimmermann. 1975. The mechanism of electrical breakdown in the membranes of Valonia utricularis. $J$. Membr. Biol. 22:73-90.

10. Neumann, E., A. E. Sowers, and C. A. Jordan. 1989. Electroporation and Electrofusion in Cell Biology. Plenum Press, New York. 436 pp.

11. Schwan, H. P. 1957. Electrical properties of tissue and cell suspensions. Adv. Biol. Med. Phys. 5:147-209.

12. Pohl, H. A. 1978. Dielectrophoresis. Cambridge University Press, London. 400 pp.

13. Kinosita, Jr. K., and T. Y. Tsong. 1978. Survival of sucrose-loaded erythrocytes in circulation. Nature (Lond.). 272:258-260.

14. Tsong, T. Y. 1987. Electric modification of membrane permeability for drug loading into living cells. Methods Enzymol. 149:248259.

15. Wong, T.-K., and E. Neumann. 1982. Electric field mediated gene transfer. Biochem. Biophys. Res. Commun. 107:584-587.

16. Neumann, E., M. Schaefer-Ridder, Y. Wang, and P. H. Hofschneider. 1982. Gene transfer into mouse lyoma cells by electroporation in high electric fields. EMBO (Eur. Mol. Biol. Organ.) J. $1: 841-845$.

17. Zimmermann, U. 1982. Electric field-mediated fusion and related electrical phenomena. Biochim. Biophys. Acta. 694:227-277.

18. Sowers, A. E. 1987. Cell Fusion. Plenum Press, New York. 540 pp.

19. Senda, M., J. Takeda, A. Shunnosuke, and T. Nakamura. 1979. Induction of cell fusion of plant protoplasts by electrical stimulation. Plant Cell Physiol. 20:1441-1443.

20. Lo, M. M. S., T. Y. Tsong, M. K. Conrad, S. M. Strittmatter, L. H. Hester, and S. H. Snyder. 1984. Monoclonal antibody production by receptor-mediated electrically induced cell fusion. $\mathrm{Na}$ ture (Lond.). 310:792-794.

21. Kanehisa, M. I., and T. Y. Tsong. 1978. Cluster model of lipid phase transitions with application to passive permeation of molecules and structure relaxations in lipid bilayers. J. Am. Chem. Soc. 100:424-432.

22. Ipsen, J. H., K. Jorgensen, and O. G. Mouritsen. 1990. Density fluctuations in saturated phospholipid bilayers increase as the acyl-chain length decreases. Biophys. J. 58:1099-1107.

23. Freire, E., and R. Biltonen. 1978. Estimation of molecular averages and equilibrium fluctuations in lipid bilayer systems from excess heat capacity function. Biochim. Biophys. Acta. 514:54-68.

24. El-Mashak, E. M., and T. Y. Tsong. 1985. Ion selectivity of temperature-induced and electric field-induced pores in dipalmitoylphosphatidylcholine vesicles. Biochemistry. 24:2884-2888.

25. Caffrey, M. 1989. The study of lipid phase transition kinetics by 
time-resolved x-ray diffraction. Annu. Rev. Biophys. Biophys. Chem. 18:159-186.

26. Tsong, T. Y., and M. I. Kanehisa. 1977. Relaxation phenomena in aqueous dispersions of synthetic lecithins. Biochemistry. 16:2674 2680.

27. Genz, A., J. F. Holzwarth, and T. Y. Tsong. 1986. The influence of cholesterol on the main phase transition of unilamellar dipalmitoylphosphatidylcholine vesicles. Biophys. J. 50:1043-1051.

28. Gruenewald, B., W. Frisch, and J. F. Holzwarth. 1981. The kinetics of the formation of rotational isomers in the hydrophobic tail region of phospholipid bilayers. Biochim. Biophys. Acta. 641:311-319.

29. Jain, K., and R. C. Wagner. 1980. Introduction to Biological Membranes. John Wiley \& Sons, New York. 382 pp.

30. Thompson, T. E., and C. Huang. 1980. Dynamics of lipids in biomembranes. In Membrane Physiology. Andreoli, T. E., J. F. Hoffman, and D. D. Fanestil, editors. Plenum Medical Book Co., New York. 27-48.

31. Tsong, T. Y., and R. D. Astumian. 1987. Electroconformational coupling and membrane protein function. Prog. Biophys. Mol. Biol. 50:1-45.

32. Benz, R., F. Beckers, and U. Zimmermann. 1979. Reversible electrical breakdown of lipid bilayer membranes: a charge-pulse relaxation study. J. Membr. Biol. 48:181-204.

33. Zimmermann, U., and R. Benz. 1980. Dependence of the electrical breakdown voltage on the charging time in Valonia utricularis. J. Membr. Biol. 53:33-43.

34. Sugar, I. P. 1983. Effect of mechanical and electrical pressure on the phase transition properties and stability of phospholipid bilayers. In Physical Chemistry of Transmembrane Ion Motions. G. Spach, editor. Elsevier, Amsterdam. 21-28.

35. Lopez, A., M. P. Rols, and J. Teissle. $1988 .{ }^{31} \mathrm{P}$ NMR analysis of membrane phospholipid organization in viable, reversibly electropermeabilized Chinese hamster ovary cells. Biochemistry. 27:1222-1228.

36. Sugar, I. P. 1989. Stochastic model of electric field-induced membrane pores. In Reference 10. 97-110.

37. Weaver, J. 1991. Electroporation. In Interfacial Phenomena in Biological Systems. M. Bender, editor. Marcel Dekker, Inc. In press.

38. Dimitrov, D. S., and A. E. Sowers. 1990. Membrane electroporation-fast molecular exchange by electroosmosis. Biochim. Biophys. Acta. 1022:381-392.

39. Tsong, T. Y. 1990. On electroporation of cell membranes and some related phenomena. Bioelectrochem. Bioenerg. 24:271-295.

40. Glaser, R. W., S. L. Leikin, L. V. Chernomordik, V. F. Pastushenko, and A. I. Sokirko. 1988. Reversible electric breakdown of lipid bilayers: formation and evolution of pores. Biochim. Biophys. Acta. 940:275-287.

41. Powell, K. T., E. G. Derrick, and J. C. Weaver. 1986. A quantitative theory of reversible electrical breakdown. Bioelectrochem. Bioenerg. 15:243-255.

42. Barnett, A., and J. C. Weaver. 1991. Electroporation: a unified, quantitative theory of reversible electrical breakdown and mechanical rupture in artificial planner bilayer membranes. Bioelectrochem. Bioenerg. In press.

43. Bliss, J. G., G. I. Harrison, J. R. Mourant, K. T. Powell, and J. C. Weaver. 1988. Electroporation: the distribution of macromolecular uptake and shape changes in red blood cells following a single $50 \mu$ s square wave pulse. Bioelectrochem. Bioenerg. 19:5771 .
44. Hartshone, R. P., B. U. Keller, J. A. Talvenheimo, W. A. Catteral, and M. Montal. 1985. Functional reconstitution of the purified brain sodium channel in planar lipid bilayers. Proc. Natl. Acad. Sci. USA. 82:240-244.

45. Tsien, R. W., P. Hess, E. W. McCleskey, and R. L. Rosenberg. 1987. Calcium channels: Mechanisms of selectivity, permeation, and block. Annu. Rev. Biophys. Biophys. Chem. 16:265-290.

46. Kim, P. S., and R. L. Baldwin. 1982. Specific intermediates in the folding reactions of small proteins and the mechanisms of protein folding. Annu. Rev. Biochem. 51:459-489.

47. Benz, R., and U. Zimmermann. 1981. The resealing process of lipid bilayers after reversible electric breakdown. Biochim. Biophys. Acta. 640:169-178.

48. Teissie, J., and T. Y. Tsong. 1981. Electric field-induced transient pores in phospholipid bilayer vesicles. Biochemistry. 20:15481554.

49. Kinosita, K., Jr., and T. Y. Tsong. 1977. Formation and resealing of pores of controlled sizes in human erythrocyte membrane. Nature (Lond.). 268:438-441.

50. Gross, D., L. M. Loew, and W. W. Webb. 1986. Optical imaging of cell membrane potential changes induced by applied electric fields. Biophys. J. 50:339-348.

51. Rosemberg, Y., and R. Korenstein. 1990. Electroporation of the photosynthetic membrane. A study by intrinsic and external optical probes. Biophys. J. 58:823-832.

52. Kinosita, K., Jr., I. Ashikava, N. Saita, H. Yoshimura, H. Itoh, K. Nagayama, and A. Ikegami. 1988. Electroporation of cell membrane visualized under pulsed laser fluorescence microscope. Biophys. J. 53:1015-1019.

53. Serpersu, E. H., K. Kinosita, Jr., and T. Y. Tsong. 1985. Reversible and irreversible modification of membrane permeability by electric field. Biochim. Biophys. Acta. 812:779-785.

54. Teissie, J., and T. Y. Tsong. 1980. Evidence of voltage induced channel opening in Na,K-ATPase of human erythrocyte membranes. J. Membr. Biol. 55:133-140.

55. Chang, D. C., and T. S. Reese. 1990. Changes of membrane structure induced by electroporation as revealed by rapidfreezing electron microscopy. Biophys. J. 58:1-12.

56. Pliquett, V. F. 1968. Das Verhalten von oxytrichiden unter einfluss des elektrischen felds. Z. Biol. 116:10-22.

57. Gass, G. V., and L. V. Chernomordik. 1990. Reversible large-scale deformations in the membranes of electrically-treated cells: electroinduced bleb formation. Biochim. Biophys. Acta. 1023:111.

58. Kinosita, K., Jr., and T. Y. Tsong. 1977. Hemolysis of human erythrocytes by a transient electric field. Proc. Natl. Acad. Sci. USA. 74:1923-1927.

59. Deuticke, B., P. Lutkemeier, and M. Sistemich. 1984. Ion selectivity of aqueous leaks induced in the erythrocyte membrane by crosslinking of membrane proteins. Biochim. Biophys. Acta. 775:150-160.

60. Schwister, K., and B. Deuticke. 1985. Formation and properties of aqueous leaks induced in human erythrocytes by electric breakdown. Biochim. Biophys. Acta. 816:332-348.

61. Schwan, H. P. 1983. Biophysics of the interaction of electromagnetic energy with cells and membranes. In Biological Effects and Dosimetry of Nonionizing Radiation. M. Grandolfo, S. M. Michaelson, and A. Rindi, editors. Plenum Press, New York. 213-231.

62. Schwan, H. P. 1989. Dielectrophoresis and rotation of cells. In 
Electroporation and Electrofusion in Cell Biology. Plenum Press, New York. 3-21.

63. Marszalek, P., D.-S. Liu, and T. Y. Tsong. 1990. Schwan equation and transmembrane potential induced by alternating electric field. Biophys. J. 58:1053-1058.

64. Chang, D. C. 1989. Cell poration and cell fusion using an oscillating electric field. Biophys. J. 56:641-652.

65. Xie, T.-D., and T. Y. Tsong. 1990. Study of mechanisms of electric field-induced DNA transfection II. Transfection by lowamplitude, low-frequency alternating electric fields. Biophys. $J$. 58:897-903.

66. Tsong, T. Y. 1974. Temperature jump relaxation kinetics of aqueous suspensions of phospholipids and $B$. subtlis membranes. Fed. Proc. 33:1342a. (Abstr.).

67. Hermann, A., M. J. Clague, A. Puri, S. J. Morris, R. Blumenthal, and S. Grimaldi. 1990. Effect of erythrocyte transbilayer phospholipid distribution on fusion with vesicular Stomatitis virus. Biochemistry. 29:4054-4058.

68. Sowers, A. E. 1986. A long-lived fusogenic state is induced in erythrocyte ghosts by electric pulses. J. Cell Biol. 102:1358-1362.

69. Schlegel, R. A., J. A. Reed, L. McEvoy, L. Algarin, and P. Williamson. 1987. Phospholipid asymmetry of loaded red cells. Methods Enzymol. 149:281-293.

70. Sowers, A. E. 1988. Fusion events and nonfusion content mixing events induced in erythrocyte ghosts by an electric pulse. Biophys. J. 54:619-626.

71. Lucy, J. A., and Q. F. Ahkong. 1988. Osmotic forces and the fusion of biomembranes. In Molecular Mechanisms of Membrane Fusion. Ohki, S., S. Doyle, T. D. Flanagan, S. W. Hui, and E. Mayhew, editors. Plenum Press, New York. 163-179.

72. Sowers, A. E., and M. L. Lieber. 1986. Electropores in individual erythrocyte ghost: diameter, lifetimes, numbers, and locations. FEBS (Fed. Eur. Biochem. Soc.) Lett. 205:179-184.

73. Kinosita, K., Jr., and T. Y. Tsong. 1977. Voltage induced pore formation and hemolysis of human erythrocyte membranes. Biochim. Biophys. Acta. 471:227-242.

74. Chernomordik, L. V., A. V. Sokolov, and V. G. Budker. 1990. Electrostimulated uptake of DNA by liposomes. Biochim. Biophys. Acta. 1024:179-183.
75. Xie, T.-D., L. Sun, and T. Y. Tsong. 1990. Study of mechanisms of electric field-induced DNA transfection I. DNA entry by surface binding and diffusion through membrane pores. Biophys. $J$. 58:13-19.

76. Mouneimne, Y., P.-F. Tosi, Y. Gazitt, and C. Nicolau. 1989. Electro-insertion of xeno-glycophorin into the red blood cell membrane. Biochem. Biophys. Res. Commun. 159:34-40.

77. Mouneimne, Y., P.-F. Tosi, R. Barhoumi, and C. Nicolau. 1990. Electroinsertion of full length recombinant CD4 into red blood cell membrane. Biochim. Biophys. Acta. 1027:53-58.

78. Tsong, T. Y. 1990. Electrical modulation of membrane proteins: enforced conformational oscillations and biological energy and signal transductions. Annu. Rev. Biophys. Biophys. Chem. 19:83106.

79. Tsong, T. Y., and R. D. Astumian. 1986. Absorption and conversion of electric field energy by membrane bound ATPases. Bioelectrochem. Bioenerg. 15:457-476.

80. Liu, D.-S., R. D. Astumian, and T. Y. Tsong. 1990. Activation of $\mathrm{Na}^{+}$and $\mathrm{K}^{+}$pumping modes of $\mathrm{Na}, \mathrm{K}$-ATPase by an oscillating electric field. J. Biol. Chem. 265:7260-7267.

81. Tsong, T. Y. 1989. Deciphering the language of cells. TIBS (Trends Biochem. Sci.). 14:89-92.

82. Blank, M. 1982. The surface compartmental model (SCM): role of surface charge in membrane permeability changes. Bioelectrochem. Bioenerg. 9:615-624.

83. Blank, M., and L. Soo. 1990. Ion activation of the Na,K-ATPase in alternating currents. Bioelectrochem. Bioenerg. 24:51-61.

84. Lee, R. C. 1990. Biophysical injury mechanisms in electrical shock victims. Proc. IEEE Eng. Med. Biol. Soc. Philadelphia. 12:15021504.

85. O'Neill, R. J., and L. Tung. 1991. A cell-attached patch clamp study of the electropermeabilization of amphibian cardiac cells. Biophys. J. 59:1028-1039.

86. Mir, L. M., S. Orlowski, J. Belehradek, Jr., and C. Paoletti. 1991. Electrochemotherapy: potentiation of antitumor effect of bleomycin by local electric pulses. Eur. J. Cancer. 27:68-72.

87. Weaver, J. C., and R. D. Astumian. 1990. The response of living cells to very weak electric fields: the thermal noise limit. Science (Wash. DC). 247:459-462. 